\title{
Oestrogen Receptor $\beta$ Activation Protects Against Myocardial Infarction via Notch1 Signalling
}

\author{
Mingjun $\mathrm{Du}^{1} \cdot$ Jianggui Shan ${ }^{1} \cdot$ Anqi Feng $^{2} \cdot$ Sebastian Schmull ${ }^{1} \cdot$ Jianmin $\mathrm{Gu}^{1,3} \cdot$ Song Xue ${ }^{1}$ (D)
}

Published online: 10 March 2020

(C) Springer Science+Business Media, LLC, part of Springer Nature 2020

\begin{abstract}
Purpose Oestrogen receptor $\beta$ is believed to exert a cardioprotective effect against ischaemic injury. Nonetheless, the mechanism underlying its protective action remains to be fully elucidated. Recently, increased attention has been focused on Notch1 signalling for ameliorating cardiac ischaemic injury. Here, we hypothesised that oestrogen receptor $\beta$ activation attenuates myocardial infarction (MI)-induced cardiac damage by modulating the Notch1 signalling pathway.

Methods Male C57BL/6 mice were used to establish an MI model through the ligation of the anterior descending branch of the left coronary artery. Two chemical drugs, 2,3-Bis(4-hydroxyphenyl)-propionitrile (DPN) and N-[N-(3,5-difluorophenacetyl)-1alanyl]-s-phenylglycine $t$-butyl ester (DAPT), a specific inhibitor of Notch1 signalling) were administered via intraperitoneal injection to change oestrogen receptor $\beta$ and Notch1 activities. Immunohistochemistry, western blot analysis, enzyme-linked immunosorbent assay (Elisa) assessment and echocardiography were used in this study to analyse cardiac oxidative stress, apoptosis, infraction volume, fibrosis and cardiac function.

Results DPN-mediated oestrogen receptor $\beta$ activation effectively protected cardiomyocytes from MI-induced oxidative damage and apoptosis. Furthermore, oestrogen receptor $\beta$ activation reduced the infarct size and lowered the levels of myocardial enzymes in the serum, thereby leading to greater overall cardiac function improvement. Ischaemic injury-induced myocardial fibrosis was attenuated by oestrogen receptor $\beta$ activation. Nevertheless, all of these cardioprotective effects of oestrogen receptor $\beta$ activation were almost abrogated by DAPT administration, i.e. DAPT attenuated the anti-oxidative and antiapoptotic effects and the decrease in infarct and fibrotic areas and reversed cardiac functional recovery. The levels of phospho-phosphatidylinositol-3-kinase (PI3K) and phospho-protein kinase B (Akt) were increased after DPN administration, and this change was reversed after DAPT was administered.

Conclusions All of these new findings indicate that oestrogen receptor $\beta$ activation is effective in ameliorating MI-induced cardiac dysfunction by enhancing Notch1 signalling and that PI3K/Akt signalling is the downstream mediator.
\end{abstract}

Keywords Oestrogen receptor $\beta$. Myocardial infarction - Notch1 signalling - Notch1 intracellular domain . Phosphatidylinositol-3-kinase · Protein kinase B (Akt)

Mingjun Du, Jianggui Shan and Anqi Feng contributed equally to this work.

Electronic supplementary material The online version of this article (https://doi.org/10.1007/s10557-020-06949-3) contains supplementary material, which is available to authorized users.

Jianmin $\mathrm{Gu}$

gujianmin@renji.com

Song Xue

xuesongrenji@163.com

1 Department of Cardiovascular Surgery, Renji Hospital, School of Medicine, Shanghai Jiao Tong University, 160 Pu-Jian Road, Shanghai 200127, People's Republic of China
2 College of Clinical Medicine, Xuzhou Medical University, Xuzhou 221004, Jiangsu, People's Republic of China

3 Department of Intensive Care Unit, Chest Hospital, School of Medicine, Shanghai Jiao Tong University, Shanghai 200030, People's Republic of China 


\section{Introduction}

Myocardial infarction (MI), which is induced by coronary atherosclerosis or spasm, usually results in cardiac dysfunction and heart failure [1]. Ischaemic cardiac tissue undergoes cardiomyocyte loss, subsequent fibrotic reparative healing and a decrease in cardiac function. The common symptom of MI is severe and persistent retrosternal pain accompanied by changes in the concentrations of serum myocardial enzymes [2]. Although many studies have focused on limiting cardiac injury after MI, few have resulted in significant clinical benefits.

Compared with men, women have a lower incidence of cardiovascular disease; this observation indicates a beneficial action of endogenous oestrogen against cardiovascular disease $[3,4]$. The effects of oestrogen are mediated mainly by oestrogen receptor $\alpha$ and oestrogen receptor $\beta$, both of which play important roles in the regulation of normal physiology and in many diseases [5]. The non-canonical effects of oestrogen are mainly mediated by G protein-coupled oestrogen receptor (GPER). Selective GPER activation has been shown to mediate beneficial effects in the cardiovascular system, as evidenced by the attenuation of the following: cardiotoxicity, MI-induced cardiac dysfunction and pulmonary hypertension, thus improving cardiovascular function in stressful conditions [6].

Oestrogen receptor $\beta$ is usually overexpressed in breast cancer, prostate cancer and other tumours, and its level changes throughout the menstrual cycle [7]. In recent years, a protective role of oestrogen receptor $\beta$ in tissue repair was revealed during ischaemic heart disease [8-10]. According to the results of studies on the development of compounds that act selectively on oestrogen receptor subtypes, various researchers claim that 2,3-bis(4-hydroxyphenyl)-propionitrile (DPN) can activate oestrogen receptor $\beta$ specifically and mediate cardioprotection after myocardial ischaemic injury [11-13]. Although the molecular mechanisms behind oestrogen receptor $\beta$-mediated protection have not yet been completely explained, there is no doubt that oestrogen receptor $\beta$ exerts a positive effect on cardiac regeneration $[14,15]$.

To date, four Notch signalling receptors (Notch1-4) and five Notch ligands (Delta-like 1, 3 and 4 and Jagged 1-2) have been identified in mammals [16]. Notch1-4 belong to a family of transmembrane receptor proteins that are pivotal components of the Notch signalling pathway. The activation of Notch signalling receptors releases a signal form of the intracellular domain of Notch (NICD). In canonical Notch signalling, the NICD is relocated to the nucleus, where it modulates the transcription of several genes by triggering allosteric changes that facilitate the displacement of transcriptional repressors and the consequent activation of downstream effector proteins [17]. The Notch1 signalling pathway has been demonstrated to perform an important function in a series of cellular processes, such as differentiation, proliferation and cell death $[18,19]$. In recent years, many studies have revealed that the Notch1 signalling pathway plays a regulatory role in cardiac diseases such as MI and heart failure [20, 21]. Although one study showed that oestrogen receptor $\beta$ dependent Notch1 activation can protect against tumour necrosis factor $\alpha$-induced vascular endothelium apoptosis, the cardioprotective effect of Notch1 via oestrogen receptor $\beta$ activation has not yet been fully characterised [22].

Therefore, on the basis of previous research, this study aimed to test the involvement of Notch1 signalling in the cardioprotective effect of oestrogen receptor $\beta$ activation. In addition, we investigated the possible involvement of downstream phosphatidylinositol-3-kinase (PI3K)/Akt signalling in this process.

\section{Materials and Methods}

\section{Reagents}

DPN, a selective oestrogen receptor $\beta$ agonist, and $N-[N-(3,5-$ difluorophenacetyl)-L-alanyl]-S-phenylglycine $t$-butyl ester (DAPT), an inhibitor of Notch, were purchased from SigmaAldrich (St. Louis, MO, USA), whereas tissue glutathione peroxidase (GPX) [23] and superoxide dismutase (SOD) activity assay kits and the malondialdehyde (MDA) assay kit were obtained from Nanjing Jiancheng Bioengineering Institute (Nanjing, Jiangsu, China). Serum creatine kinase (CK), creatine kinase MB form (CK-MB) and lactate dehydrogenase (LDH) assay kits were purchased from Changchun Huili Bio-tech Co., Ltd. (Changchun, Jilin, China), and the serum cardiac troponin $\mathrm{T}$ (cTnT) enzyme-linked immunosorbent assay (Elisa) kit was obtained from CUSABIO (Wuhan, Hubei, China). Anti-Notch1 intracellular domain (NICD), anti-oestrogen receptor $\beta$, anti-inducible nitric oxide synthase (iNOS), anti-vascular endothelial growth factor (VEGF), anti-endothelial nitric oxide synthase (eNOS) and anti-Bcl-2 antibodies were acquired from Abcam (Cambridge, UK), whereas anti-caspase-3, antiphospho-(p-)PI3K, anti-PI3K, anti-p-Akt, anti-Akt and antirabbit IgG antibodies were acquired from Cell Signaling Technology (Beverly, MA, USA). The terminal deoxynucleotidyl transferase-mediated dUTP-biotin nick-end labelling (TUNEL) assay kit was purchased from Roche Life Science (Indianapolis, IN, USA) and the 3,3'-diaminobenzidine tetrahydrochloride (DAB) kit was purchased from Vector Laboratories, Inc. (Burlingame, CA, USA). 2,3,5-Triphenyltetrazolium chloride (TTC) was acquired from Sigma-Aldrich.

\section{Animals}

All procedures in this study, including animal use, housing and surgical procedures, were approved by the ethics committee of Renji Hospital (Shanghai, China) and conformed to the Guide for the Care and Use of Laboratory Animals published by the 
National Health and Family Planning Commission of the People's Republic of China. C57BL/6 mice (males; weight range, 20-25 g) were obtained from Shanghai Laboratory Animal Center, the Chinese Academy of Sciences (SLACCAS, Shanghai, China). All animals were housed on a 12-h light/12-h dark cycle with a controlled temperature range $\left(20-24{ }^{\circ} \mathrm{C}\right)$ and relative humidity range (40-70\%). All mice had free access to food and water.

\section{Construction of a Mouse Model of MI}

To induce MI, mice were anaesthetised by inhalation of $3 \%$ isoflurane in a chamber. A rodent ventilator (model 683, Harvard Apparatus, Inc., Holliston, MA, USA) was used with $1.5 \%$ isoflurane during the surgical procedure [24]. The depth of anaesthesia was deemed adequate when there were neither motor nor autonomic responses to nose pinching. The mice were kept warm with heat lamps and heating pads. The chest was opened with a horizontal incision through the muscle between the ribs at the third intercostal space, and ischaemia was implemented by ligating the anterior descending branch of the left coronary artery using a 7-0 nylon suture. After that, the incision was closed, and the wound was cleaned and disinfected. Shamoperated mice underwent the same procedure, with the exception of the left coronary artery ligation. Functional observations (respiration, heartbeat and neural reflexes) were performed daily on all mice after the operation [25].

\section{Experimental Protocols}

C57BL/6 mice were randomly assigned to the following five groups (each $n=6$ ): (1) sham + saline (mice that received saline by intraperitoneal injection 14 days before the sham operation and 2 days after the sham operation), (2) MI + saline (mice that received saline by intraperitoneal injection 14 days before MI and 2 days after MI), (3) MI + DPN (mice that received $0.8 \mathrm{mg} / \mathrm{kg}$ per day DPN by intraperitoneal injection 14 days before MI and 2 days after MI), (4) MI + DPN + DAPT (mice that received $0.8 \mathrm{mg} / \mathrm{kg}$ per day DPN and $10 \mathrm{mg} / \mathrm{kg}$ per day DAPT by intraperitoneal injection 14 days before MI and 2 days after MI and (5) MI + DAPT (mice that received $10 \mathrm{mg} / \mathrm{kg}$ per day DAPT by intraperitoneal injection 14 days before MI and 2 days after MI). Timeline of experimental protocols was presented in SFig. 1. DPN and DAPT were initially dissolved in dimethyl sulfoxide and then diluted in sterile saline (final dimethyl sulfoxide concentration $<2 \%$ ). The doses of DPN and DAPT were chosen according to previous studies [13, 26, 27]. Post-infarction remodelling has been peremptorily divided into an early phase (within $72 \mathrm{~h}$ ) and a late phase. In the early phase post-MI, we examined the oxidative stress, the number of apoptotic cells, the myocardial enzyme release and the cardiac-infarcted volume. In the late phase, we evaluated the cardiac fibrotic areas and the heart function [28]. We executed the animals by using carbon dioxide anaesthesia. When the mice were anesthetised, they were killed by cervical dislocation. Of note, to avoid causing pain, the flow rate of carbon dioxide was $10-30 \%$ of the chamber volume per minute. Once each assessed mouse was killed, the hearts were carefully removed [25]. For western blot analysis and Elisa, the whole heart tissue was collected and washed with phosphate-buffered saline (PBS) to flush out residual blood before homogenisation. For histological assessment and infarct size measurement, heart ventricles from the ligature to the apex were rapidly isolated and rinsed thoroughly with PBS before being fixed or sliced.

\section{Western Blot Analysis}

Cardiac tissues (3 days after MI or sham operation) were homogenised in radio immunoprecipitation assay (RIPA) lysis buffer (Sigma-Aldrich) with protease and phosphatase inhibitor mixtures (Roche Life Science). Protein concentrations were measured with the bicinchoninic acid (BCA) protein assay kit (Pierce Biotechnology, Rockford, AL, USA). An equal amount of protein samples (50 $\mu \mathrm{g}$ protein) from each group was separated by $4-12 \%$ SDS-PAGE (Beyotime, Shanghai, China) and transferred onto polyvinylidene difluoride membranes. The blots were incubated overnight at $4{ }^{\circ} \mathrm{C}$ with antibodies against NICD, oestrogen receptor $\beta$, Bcl-2, cleaved caspase-3, eNOS, p-PI3K, PI3K, p-Akt, Akt or GAPDH. The membranes were washed and incubated with a goat anti-rabbit IgG antibody for $1 \mathrm{~h}$ at room temperature. Finally, the bands were developed with the enhanced chemiluminescence substrate kit (Millipore, Billerica, MA, USA). Quantification of the blots was performed with the ImageJ software, version 1.8.0.

\section{Immunofluorescence}

Three days after MI induction or sham operation, murine heart specimens were fixed in $10 \%$ formalin, incubated in a graded series of ethanol solutions and xylene and then embedded in paraffin. Cardiac tissues were sectioned at a thickness of $5 \mu \mathrm{m}$. The tissue sections were blocked in $3 \%$ bovine serum albumin for $1 \mathrm{~h}$ at room temperature (RT). Then, the sections were incubated in PBS containing 0.3\% Triton X-100 and a rabbit anti-NICD antibody (1:200 dilution) at $4{ }^{\circ} \mathrm{C}$ overnight. The sections were then incubated with an Alexa Fluor $488_{-}$ conjugated goat anti-rabbit IgG secondary antibody at room temperature in the dark for $1 \mathrm{~h}$ and with blue nuclear 4,6diamidino-2-phenylindole (DAPI) for $1 \mathrm{~min}$. Images were obtained using fluorescent microscopy (Nikon, Tokyo, Japan).

\section{Immunohistochemistry}

For immunohistochemistry staining, the sections were exposed to $3 \%$ hydrogen peroxide for $10 \mathrm{~min}$ to suppress 
endogenous peroxidase activity and then blocked with $3 \%$ bovine serum albumin for $30 \mathrm{~min}$ at RT. The tissue sections were then incubated overnight at $4{ }^{\circ} \mathrm{C}$ in PBS containing $0.3 \%$ Triton X-100 and a rabbit anti-mouse iNOS antibody (1:200 dilution) or a rabbit anti-mouse VEGF antibody (1:200 dilution). The sections were then incubated with an anti-rabbit IgG secondary antibody for $1 \mathrm{~h}$ at room temperature. After washing in PBS, immunoreactivity was detected with the DAB kit, and the sections were counterstained with haematoxylin. Finally, the tissue sections were dehydrated in a graded ethanol series and were coverslipped. The results were examined under a DP70 microscope (Olympus, Inc., Tokyo, Japan).

\section{Oxidative Stress Measurement}

Three days after MI induction, murine hearts were collected, rinsed thoroughly with PBS, and homogenised. The homogenates were centrifuged at $400 \times \mathrm{g}$ for $30 \mathrm{~min}$, and the supernatants were collected and stored at $-70{ }^{\circ} \mathrm{C}$ until analysis. Oxidative stress in the murine heart was evaluated by assessing the concentrations of MDA, GPX and SOD with the commercial kits mentioned above.

\section{TdT-Mediated dUTP Nick-End Labelling Assay}

Mice were euthanised 3 days after MI induction. Cardiac sections were prepared as described above. TUNEL assays were performed with the TUNEL assay kit (Roche Life Science). In five randomly chosen visual fields at high magnification, TUNEL-positive cells that exhibited green nuclear staining and all cells stained with DAPI were counted. Images were captured and quantitatively analysed with a DP70 microscope (Olympus, Tokyo, Japan).

\section{Infarct Size Measurement}

Infarct size was determined on day 3 after MI induction. Whole heart tissue was removed and sliced into $\sim 2$-mm transverse sections, which were then placed in $1 \%$ TTC solution for $30 \mathrm{~min}$ at $37^{\circ} \mathrm{C}$ [29]. The infarcted area of the tissue slices was measured with the ImageJ software, version 1.8.0.

\section{Myocardial Damage Assessment}

Myocardial damage was evaluated by measuring the levels of myocardial enzymes in the blood serum. Three days after MI induction, blood was collected from the carotid artery and incubated at room temperature for $30 \mathrm{~min}$. Next, the serum was separated by centrifugation $(400 \times g$ for $30 \mathrm{~min}$ at room temperature) and stored at $-70{ }^{\circ} \mathrm{C}$. CK, CK-MB, LDH and cTnT levels were measured using the aforementioned commercial kits.

\section{Echocardiography}

Transthoracic echocardiography was conducted to assess cardiac function on days 3 and 28 after the surgical procedure using a Vevo 2100 high-resolution imaging system. The mice were anaesthetised via $1.5 \%$ isoflurane inhalation and placed in the supine position. The chest fur was removed with a depilatory cream. Two-dimensional echocardiographic images and M-mode traces were acquired in the parasternal short-axis view at the level of the papillary muscles. To evaluate ventricular volume changes, the left ventricle (LV) end-diastolic volume (EDV) and LV endsystolic volume (ESV) were measured. Moreover, systolic function was measured by calculating the ejection fraction (EF).

\section{Myocardial Fibrosis}

Twenty-eight days after MI induction, cardiac sections were prepared as described above. Heart sections were stained with haematoxylin and eosin (H\&E), Sirius Red or Masson's trichrome as indicators of myocardial fibrosis [30, 31]. The thickness of the left ventricular wall was examined by measuring the average thickness of the LVs. Scar size was evaluated by calculating the percentage of the viable red or Masson's trichrome area relative to the entire area with the ImageJ software, version 1.8.0.

\section{Statistical Analysis}

The results were presented as the mean \pm standard error of the mean (SEM). Each experiment was repeated at least three times. One-way analysis of variance with Tukey's post hoc test was carried out for multiple comparisons. $P<0.05$ was assumed to indicate a statistically significant difference. The SPSS 16.0 software (SPSS Inc., Chicago, IL, USA) was employed for the statistical analysis.

\section{Results}

\section{The Expression of Oestrogen Receptor $\beta$ and NICD in the Heart After Different Treatments}

To our knowledge, there are currently no data in the literature regarding the expression of oestrogen receptor $\beta$ in the heart after an ischaemic insult. In our experiments, we demonstrated that there was no significant difference in cardiac oestrogen receptor $\beta$ expression among the sham + saline, $\mathrm{MI}+$ saline and $\mathrm{MI}+\mathrm{DPN}$ groups $(P>0.05$; Fig. 1a, b).

The expression of NICD represents the activation of Notch1 signal transduction. We examined cardiac NICD 
a

ER $\beta$

GAPDH
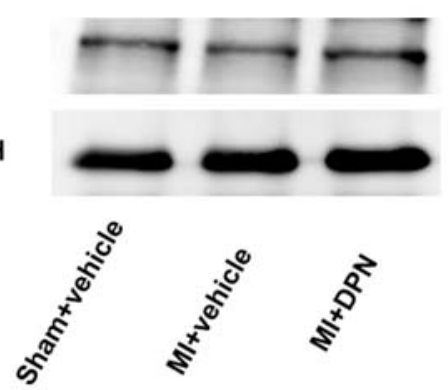

b

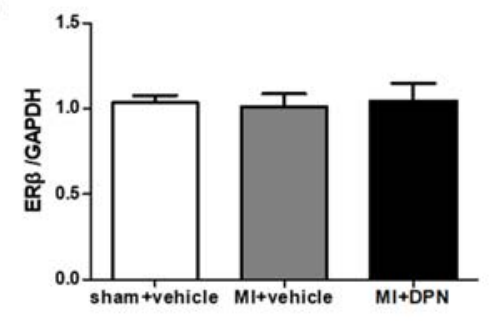

C

NICD

DAPI

merge
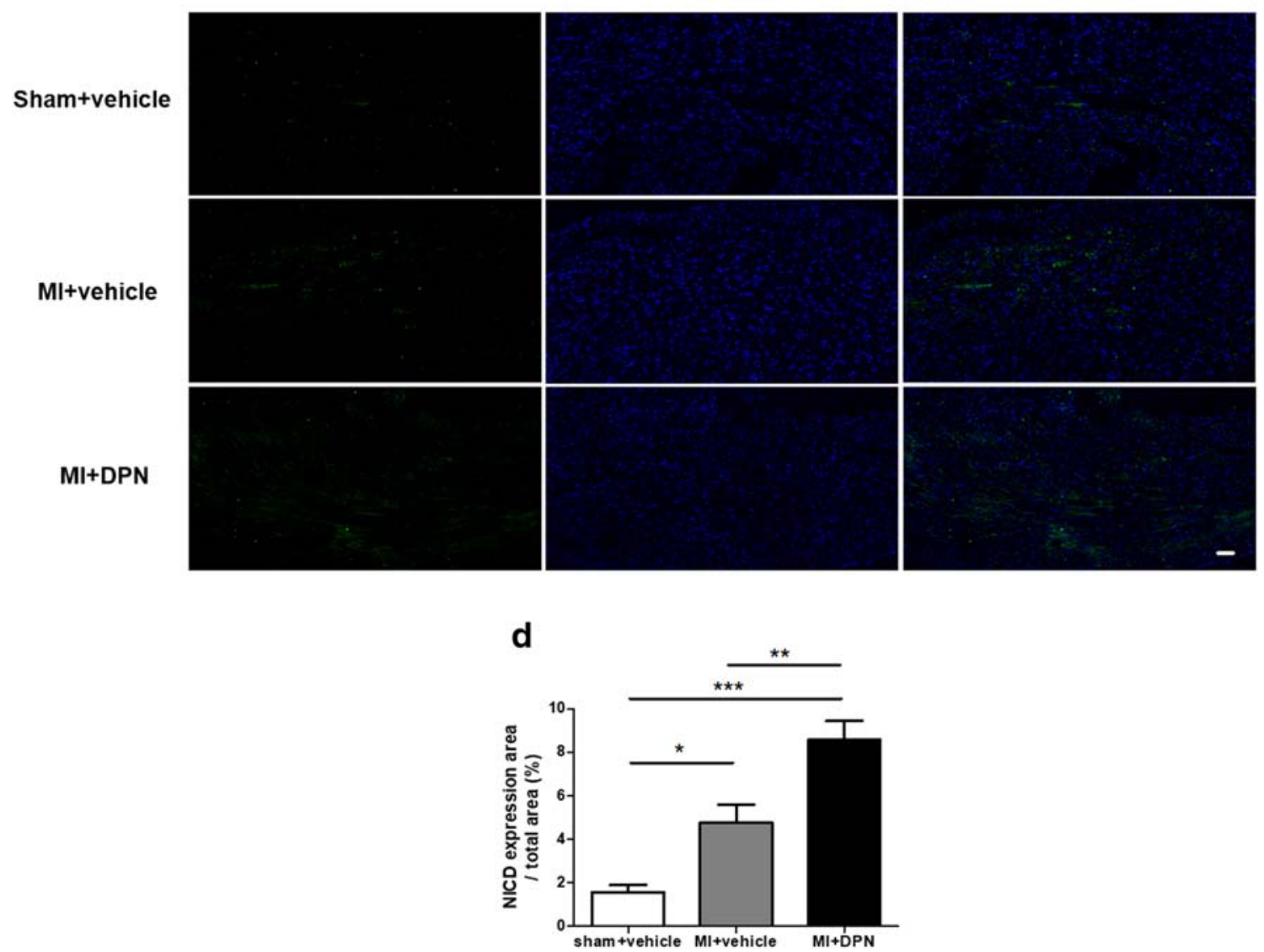

Fig. 1 The expression of oestrogen receptor $\beta$ and intracellular domain of Notch (NICD) in the murine heart. a Cardiac oestrogen receptor $\beta$ levels, as measured by western blotting $(n=3)$. b Assessment of oestrogen receptor $\beta$ expression. c Cardiac NICD expression, as evaluated by

immunofluorescence analysis (scale bar, $50 \mu \mathrm{m} ; n=6$ ). d Analysis of NICD expression. The values are the means \pm SEM. $* P<0.05$, $* * P<0.01$ and $* * * P<0.001$

expression by immunofluorescence analysis (Fig. 1c). The immunofluorescence results showed that the endogenous cardiac Notch1 was activated significantly in the $\mathrm{MI}+$ saline and MI + DPN groups compared with that in the sham + saline group $(P<0.05$ and $P<0.001$, respectively). Moreover, NICD was more upregulated in the MI + DPN group than in the MI + saline group $(P<0.01$; Fig. 1c, d).

\section{The Protective Action of Oestrogen Receptor $\beta$ (by Activating Notch1) Against Myocardial Oxidative Damage}

Oxidative stress is known to exert a strong influence on the pathophysiology of MI. Therefore, we examined the levels of several markers of oxidative stress in the myocardium 3 days after MI induction. Immunohistochemical results suggested that 
the expression of iNOS, a marker of oxidative stress, was effectively attenuated in the MI + DPN group compared with that in the other groups $(P<0.01$ compared with the $\mathrm{MI}+$ saline, $\mathrm{MI}+$ DPN + DAPT and MI + DAPT groups; Fig. 2a, b). Furthermore, it has been reported that selenoproteins seem to be indispensable for cardioprotection in oxidative injury $[32,33]$. Therefore, the concentration of MDA and the activities of GPX and SOD were examined in our study. Our results showed that DPN treatment attenuated the MI-induced elevation of the MDA level $(P<0.05$ compared with the MI + saline and MI + DAPT groups; Fig. 2c). Furthermore, SOD was upregulated in the MI + DPN group $(P<0.01$ compared with the $\mathrm{MI}+$ saline group $)$, whereas the DAPT treatment almost completely abrogated these effects of DPN, as observed in the MI + DPN + DAPT group $(P<0.05$; Fig. 2d). Similarly, GPX expression was higher in the MI + DPN group than in the $\mathrm{MI}+$ saline and $\mathrm{MI}+\mathrm{DAPT}$ groups $(P<0.05$
Fig. 2 Effects of 2,3-bis(4hydroxyphenyl)-propionitrile (DPN) and $N-[N-(3,5-$ difluorophenacetyl)-1-alanyl]-sphenylglycine $t$-butyl ester (DAPT) on cardiomyocytic oxidative damage after myocardial infarction (MI). a and b Representative images of immunohistochemical staining and the analysis of inducible nitric oxide synthase (iNOS) expression in mouse myocardial sections from different groups (scale bar, $50 \mu \mathrm{m})$. c-e Levels of malondialdehyde (MDA), superoxide dismutase (SOD) and glutathione peroxidase (GPX) were assessed in myocardial tissues. The data are the means \pm SEM. $n=6 ; * P<0.05$ and $* * P<0.01$ a

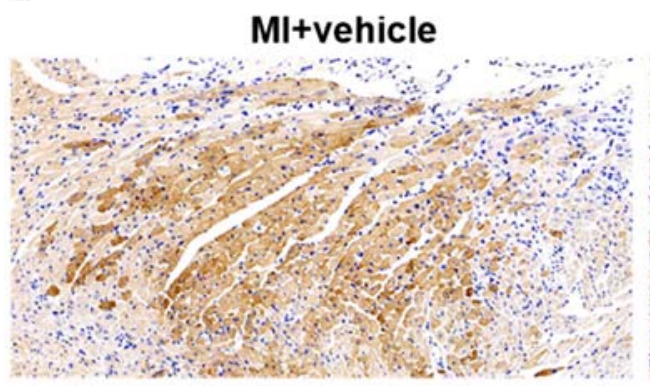

MI+DPN+DAPT

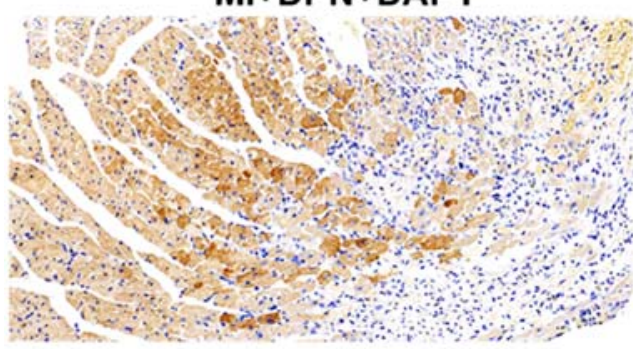

b

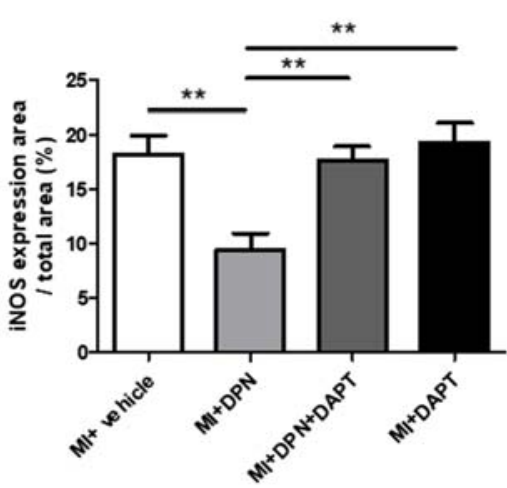

d

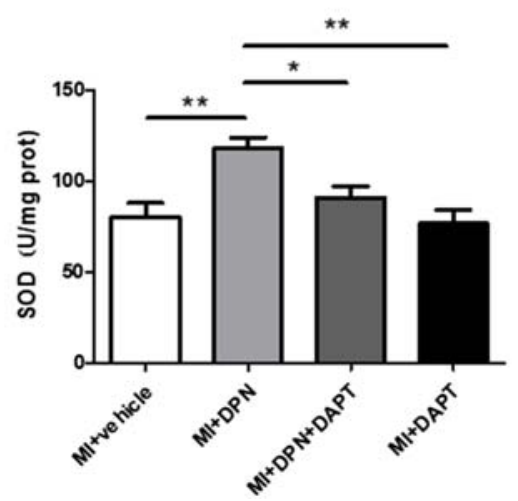

MI+DPN

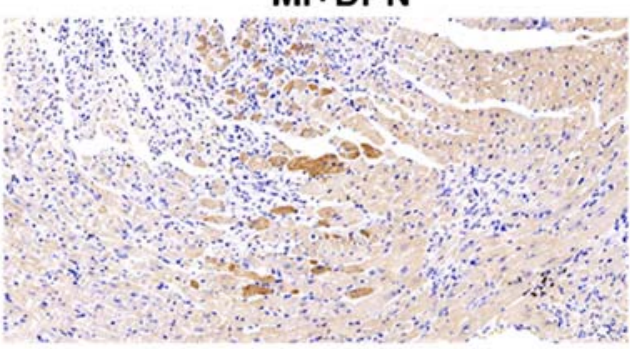

MI+DAPT

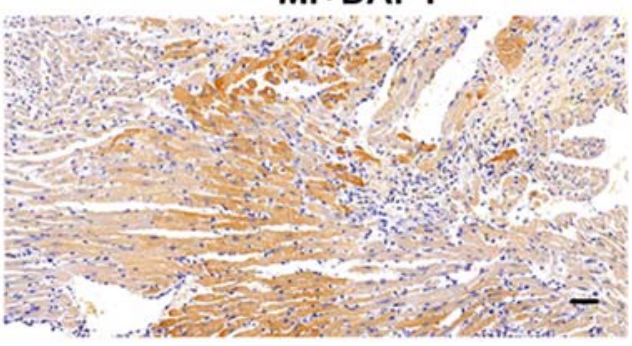

C

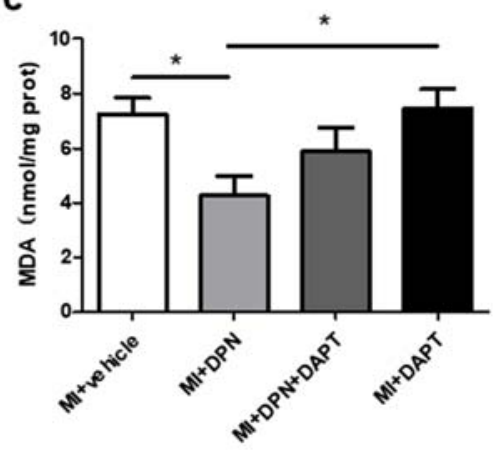

e

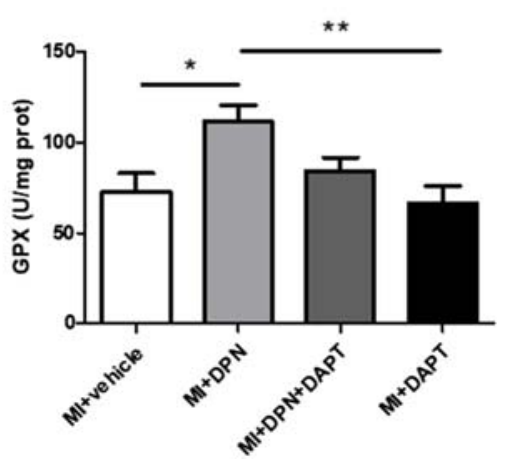


in comparison with the $\mathrm{MI}+$ saline group and $P<0.01$ compared with the MI + DAPT group; Fig. 2e).

\section{Oestrogen Receptor $\beta /$ Notch1 Signalling Protects the Myocardium from Apoptosis Caused by MI}

Myocardial apoptosis induced by MI is a major cause of cardiac functional impairment. To investigate the specific role of oestrogen receptor $\beta /$ Notch1 signalling in MI injury, we measured myocardial apoptosis (by the TUNEL assay; Fig. 3a) and the expression of cleaved caspase-3 and Bcl-2 (Fig. 3b). The results of the TUNEL assay indicated that DPN treatment significantly reduced myocardial apoptosis after MI $(P<0.01$; Fig. 3c). In contrast, the inhibition of NICD by DAPT attenuated the antiapoptotic influence of oestrogen receptor $\beta$ activation $(P<0.01$ compared with the MI + DPN group; Fig. 3c). Similarly, the apoptosis-related protein Bcl-2 was more strongly expressed in the MI + DPN group than in the other groups $(P<0.01$; Fig. $3 \mathrm{~d}$ ), and cleaved caspase- 3 was more effectively repressed in the $\mathrm{MI}+\mathrm{DPN}$ group compared with that in the MI + saline $(P<0.05$; Fig. 3e) and MI + DAPT groups ( $P<0.05$; Fig. 3e).

\section{Oestrogen Receptor $\beta$ Stimulation (by Activating Notch1) Reduced the Infarct Size and Attenuated Myocardial Damage in MI Mice}

To further assess the participation of oestrogen receptor $\beta$ in cardiac repair, we examined the infarct size in hearts from MI mice subjected to different treatments (Fig. 4a). Notably, in the MI + DPN group, a significantly reduced infarct size was observed compared with that in the other groups $(P<0.01$ compared with the MI + saline and MI + DAPT groups and $P<0.05$ in comparison with the MI + DPN + DAPT group; Fig. 4b). Moreover, cardiac damage was evaluated by measuring the release of several markers (CK, CK-MB, LDH and cTnT) into the blood serum. A similar trend among these serum markers was revealed. Our analysis indicated that the MI + DPN treatment significantly decreased the serum levels of CK, CK-MB, cTnT and LDH in comparison with groups $\mathrm{MI}+$ saline, $\mathrm{MI}+\mathrm{DPN}+$ DAPT and MI + DAPT $(P<0.05$ or $P<0.01 ;$ Fig. $4 \mathrm{c}-\mathrm{f})$.

\section{Oestrogen Receptor $\beta /$ Notch1 Signalling Increases the Expression of VEGF and eNOS in the Cardiac Tissues After MI}

VEGF and eNOS are considered to be important regulators for myocardial angiogenesis after MI. As shown in SFig. 2 a and $\mathrm{b}, \mathrm{MI}$ significantly increased the levels of VEGF in the MI + DPN group compared with that in the $\mathrm{MI}+$ vehicle $(P<0.01)$ and $\mathrm{MI}+\mathrm{DAPT}$ groups $(P<0.001)$. Furthermore, the western blot results showed that the eNOS expression was upregulated significantly in the MI + DPN group than in the MI + vehicle and MI + DAPT groups $(P<0.05$; SFig. 2c, d).

\section{Oestrogen Receptor $\beta /$ Notch1 Signalling Restored Cardiac Function After MI}

Echocardiography was performed on days 3 and 28 after MI induction (Fig. 5a). Three days after MI, the MI + DPN group manifested better cardioprotection than the other groups in terms of EF $(P<0.01$ compared with the $\mathrm{MI}+\mathrm{DPN}+$ DAPT group and $P<0.001$ compared with the $\mathrm{MI}+$ saline and MI + DAPT groups), EDV $(P<0.05$ relative to the MI + $\mathrm{DPN}+\mathrm{DAPT}$ group and $P<0.01$ compared with the $\mathrm{MI}+$ saline and MI + DAPT groups) and ESV $(P<0.05$ compared with the MI + DPN + DAPT group and $P<0.001$ in comparison with the $\mathrm{MI}+$ saline and MI + DAPT groups). Nevertheless, no significant differences were observed even though the MI + DPN + DAPT group seemed to show slightly better cardiac function than the MI + saline and MI + DAPT groups in terms of EF, EDV and ESV (Fig. 5b-d). On day 28, the reductions in EDV and ESV were associated with increased EF values in the MI + DPN group. The mice in the $\mathrm{MI}+\mathrm{DPN}$ group had significantly higher EF values compared with those of the mice in the MI + saline $(P<0.01), \mathrm{MI}+\mathrm{DPN}$ + DAPT $(P<0.05)$ and MI+ DAPT groups $(P<0.001)$. In addition, the MI + DPN group had significantly lower EDV and ESV values compared with those of the MI + saline $(P<0.05$ for EDV and $P<0.01$ for ESV $)$ and $\mathrm{MI}+\mathrm{DAPT}$ groups $(P<0.01$; Fig. 5c, d). Nonetheless, no significant differences in EDV and ESV values were observed between the $\mathrm{MI}+\mathrm{DPN}$ and MI + DPN + DAPT groups (Fig. 5c, d). In brief, these findings suggested that DPN treatment without DAPT administration moderately attenuated post-MI LV function. DPN + DAPT treatment was not as effective as the administration of DPN, only the administration in protecting cardiac function post-MI.

\section{Oestrogen Receptor $\beta$-Dependent Notch1 Signalling Restrains LV Remodelling}

To assess the remodelling of LVs and histological changes after MI, H\&E staining was performed 4 weeks after MI induction (Fig. 6a). After MI, a thin expanded wall appeared in the LVs. The MI + DPN group showed thicker LV walls in the surviving myocardium in comparison with those shown by the other groups $(P<0.05$ compared with the $\mathrm{MI}+\mathrm{DPN}+\mathrm{DAPT}$ group, $P<0.001$ compared with the $\mathrm{MI}+$ saline group and $P<0.001$ relative to the MI + DAPT group). Moreover, the average thickness of the LVs was even lower in the MI + DAPT group than in the MI + DPN + DAPT group $(P<0.05$; Fig. 6b).

MI injury often causes fibrotic area formation due to cardiomyocyte death and myofibroblast recruitment. Thus, Sirius Red staining and Masson's trichrome staining were performed 4 weeks after MI induction to measure the fibrotic areas. As depicted in Fig. 6a, a large quantity of collagen was deposited 
Fig. 3 Action of 2,3-bis(4hydroxyphenyl)-propionitrile (DPN) and $N-[N-(3,5-$ difluorophenacetyl)-1-alanyl]-sphenylglycine $t$-butyl ester (DAPT) on cardiomyocyte apoptosis post-MI. a TUNEL staining was performed on left ventricle (LV) tissues from different treatment groups (scale bar, $50 \mu \mathrm{m})$. b Cardiac Bcl-2 and cleaved caspase-3 levels, as measured by western blotting. c-e The quantification of TUNEL staining $(n=6)$ and Bcl-2 and cleaved caspase- 3 expression $(n=3)$. The values are presented as the means \pm SEM. $* P<0.05$, $* * P<0.01$ and $* * * P<0.001$ a
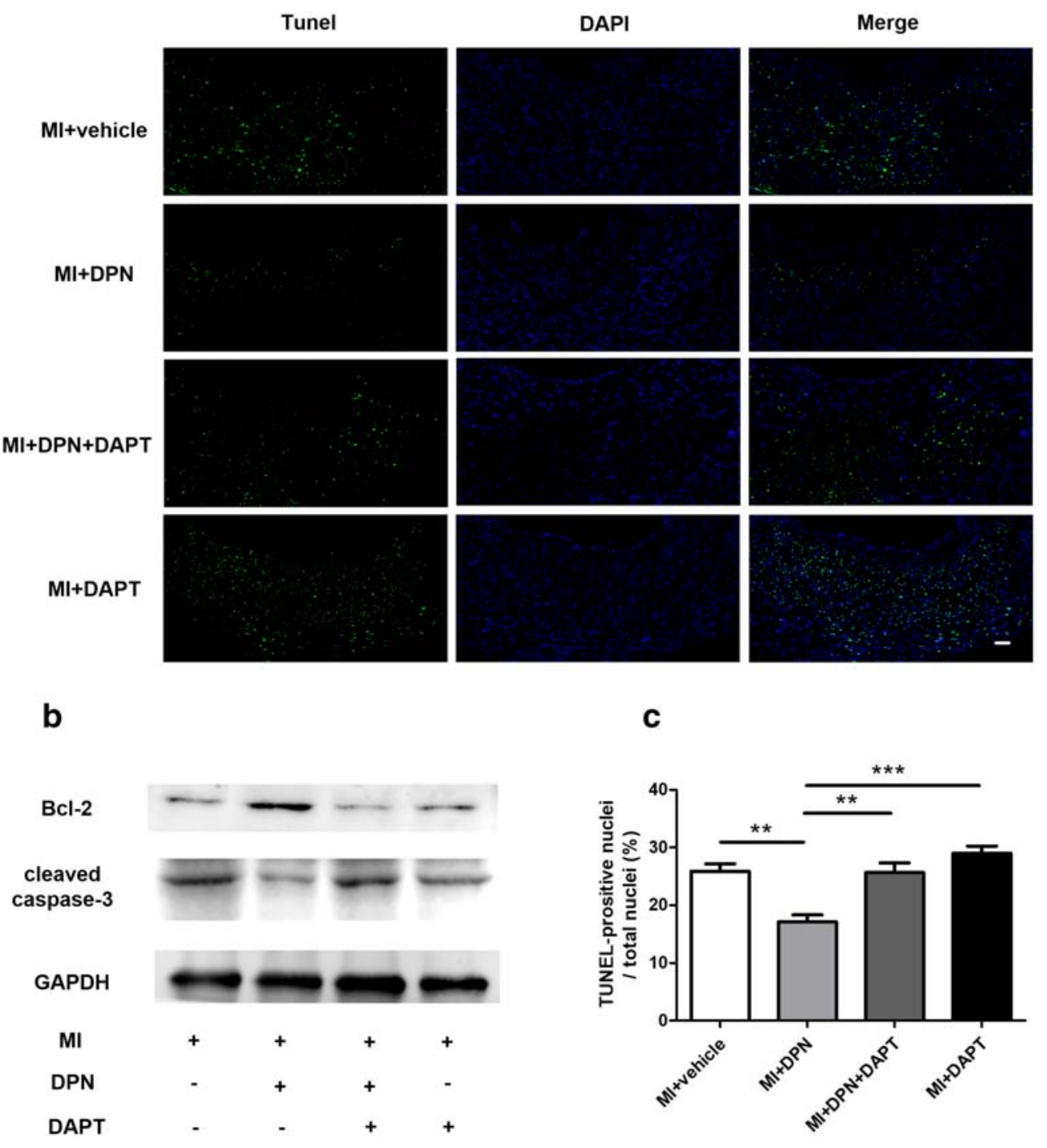

d

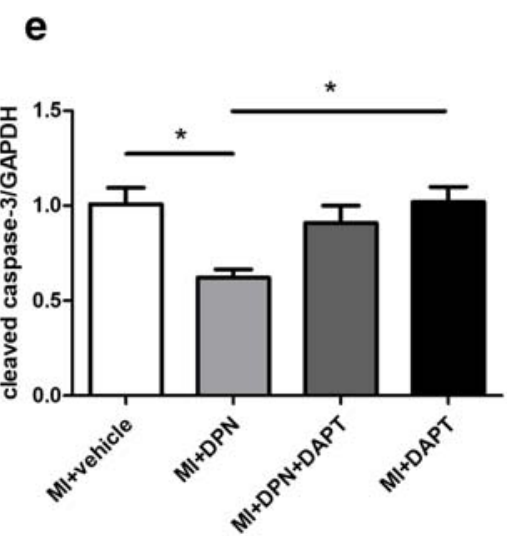

in the infarct area, and surviving myocardium was hardly detectable in the MI + saline, $\mathrm{MI}+\mathrm{DPN}+\mathrm{DAPT}$ and $\mathrm{MI}+$ DAPT groups. In contrast, in the MI + DPN group, there was clearly less collagen hyperplasia, and a large proportion of unaffected myocardium was observed $(P<0.05$ compared with the MI + DPN + DAPT group, $P<0.01$ compared with the MI + saline group and $P<0.001$ compared with the MI + DAPT group; Fig. 6c). In addition, the fibrotic area of the LVs was even larger in the MI + DAPT group than in the MI + DPN + DAPT group $(P<0.05$; Fig. 6c) . 


\section{Oestrogen Receptor $\beta$ Activation Exerts}

its Cardioprotective Function

Through the Notch1/PI3K/Akt Signalling Cascade

To test whether the PI3K/Akt pathway is involved in the molecular mechanisms of oestrogen receptor $\beta /$ Notch1 signallingmediated cardioprotection, we assessed the levels of NICD, p$\mathrm{PI} 3 \mathrm{~K}$ and $\mathrm{p}$-Akt in cardiac tissues 3 days after MI induction (Fig.7a). The results revealed that treatment with DPN significantly increased the protein expression of NICD $(P<0.01)$ and the phosphorylation of PI3K $(P<0.001)$ and Akt $(P<0.001)$ relative to the levels in the $\mathrm{MI}+$ saline group (Fig. $7 \mathrm{~b}-$ d). Nonetheless, these phenomena were almost completely abrogated by DAPT treatment $(P<0.01$ or $P<0.001)$. In addition, there were no significant differences in NICD expression between the MI + DPN + DAPT and MI + DAPT groups (Fig. 7b). Finally, no significant difference in total PI $3 \mathrm{~K}$ and Akt expression after different treatments was observed $(P>0.05 ;$ Fig. 7 e, f).
Fig. 4 Effects of 2,3-bis(4hydroxyphenyl)-propionitrile (DPN) and $N-[N-(3,5-$

difluorophenacetyl)-1-alanyl]-sphenylglycine $t$-butyl ester (DAPT) on cardiac infarct size and the release of myocardial enzymes. $\mathbf{a}$ and $\mathbf{b}$ TTC staining of transverse sections and the quantification of the infarct size in each group. $\mathbf{c}-\mathbf{f}$ Total creatine kinase $(\mathrm{CK})$, creatine kinase $\mathrm{MB}$ form (CK-MB), lactate dehydrogenase (LDH) and cardiac troponin $\mathrm{T}(\mathrm{cTnT})$ levels in the arterial blood serum. The values are the means \pm SEM. $n=$ $6 ; * P<0.05$ and $* * P<0.01$ a Ml+vehicle
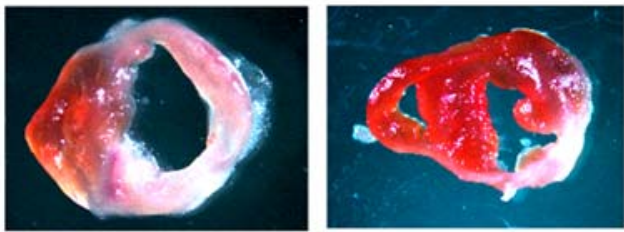

b

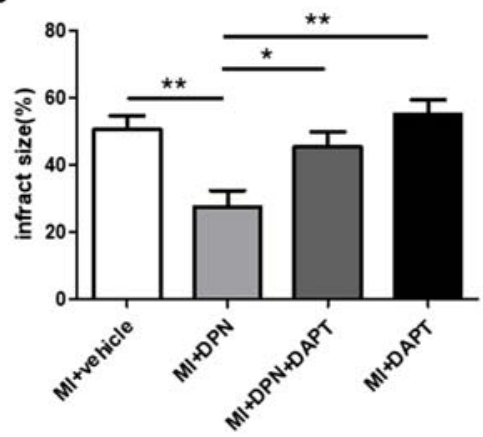

d

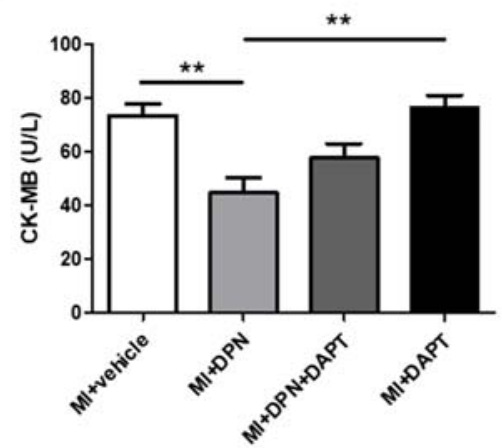

f

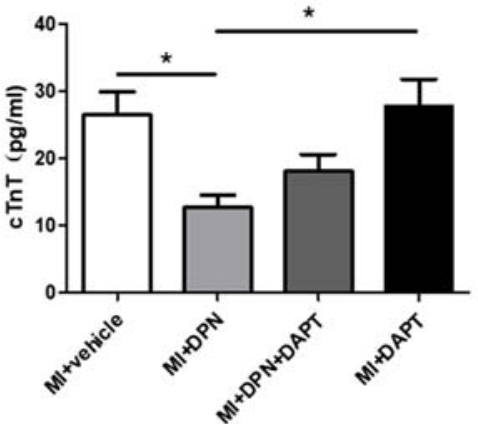

\section{MI+DPN+DAPT}

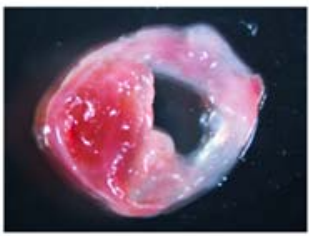

C

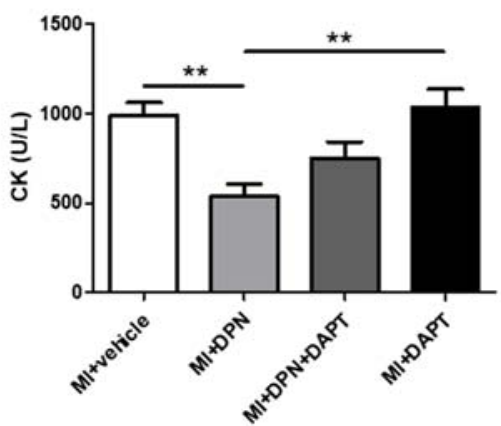

e

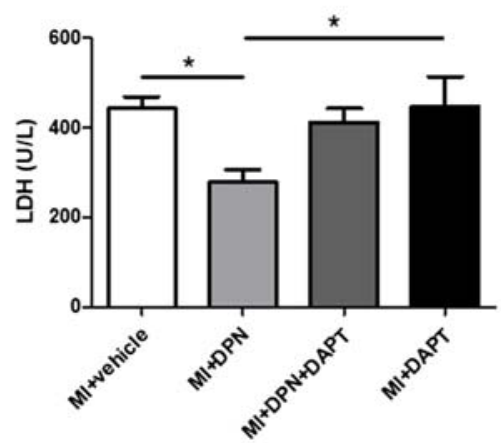




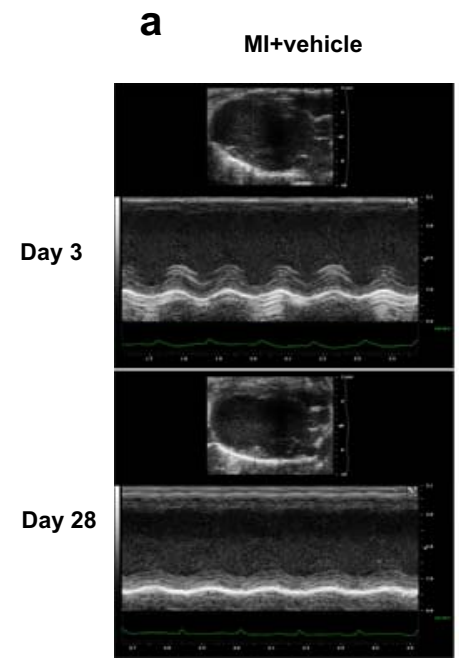

b

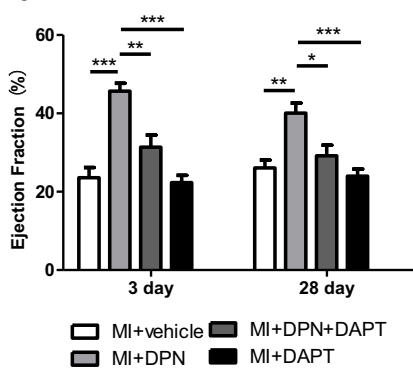

MI+DPN

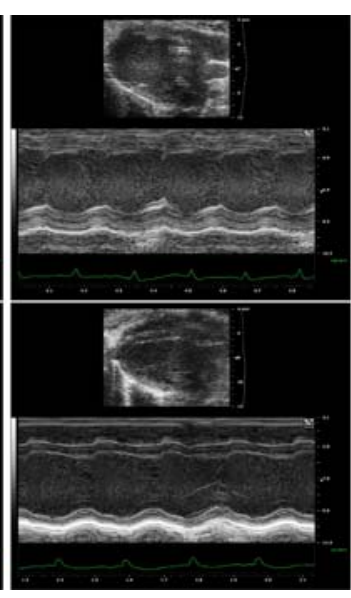

MI+DPN+DAPT

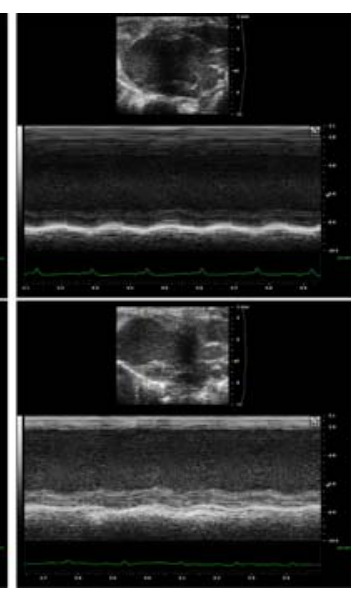

MI+DAPT

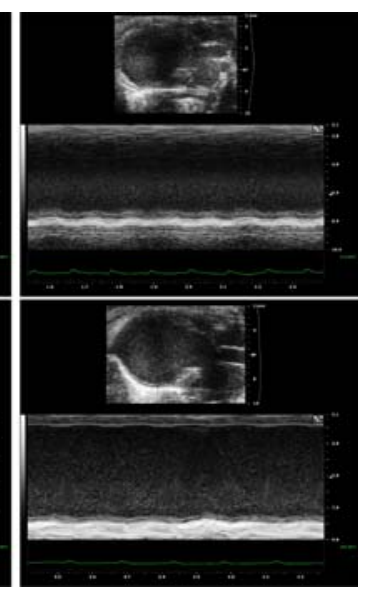

C

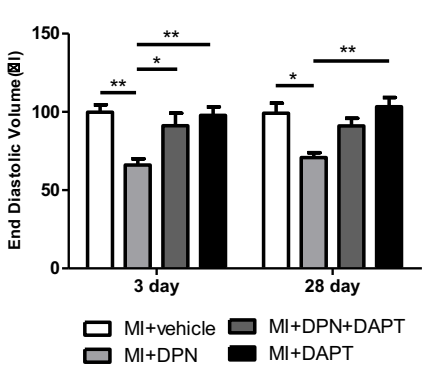

d

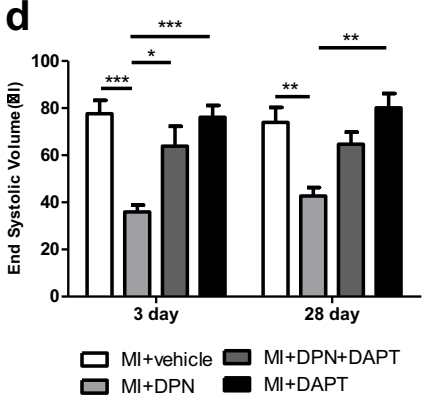

Fig. 5 Influence of 2,3-bis(4-hydroxyphenyl)-propionitrile (DPN) and $N$ [ $N$-(3,5-difluorophenacetyl)-1-alanyl]-s-phenylglycine $t$-butyl ester (DAPT) on cardiac function after MI. a M-mode images revealing cardiac function. b-d The ejection fraction (EF), end-diastolic volume

\section{Discussion}

The results of our experiments using a specific oestrogen receptor $\beta$ agonist and a Notch1 inhibitor clearly showed that direct oestrogen receptor $\beta$ activation protects the heart against oxidative stress, cardiomyocyte apoptosis, cardiac fibrosis and the deterioration of cardiac function post-MI and that Notch1 signalling may play a pivotal role in this process. Further examination of the mechanism indicated that these phenomena are mediated by the PI3K/Akt pathway (Fig. 8).

Oestrogen receptor $\beta$ initially attracted attention due to its association with the health benefits of oestrogen and its effects in cancer [34]. Studies in recent years have revealed the protective effect of oestrogen against multiple cardiovascular diseases, and further investigation has proven that the protective function of oestrogen can be mainly attributed to its receptors (oestrogen receptor $\alpha$ and oestrogen receptor $\beta$ ) $[35,36]$. The cardioprotective actions of oestrogen receptor $\beta$, particularly in females, have been reported in other studies and involve the ability to activate the NO pathway in endothelial cells, reduce oxidative stress, alleviate vascular inflammation and promote angiogenesis [37]. Many current studies use oestrogen receptor
(EDV) and end-systolic volume (ESV) were measured 3 and 28 days after MI in the different groups. The data are the means \pm SEM. $n=6$; $* P<0.05, * * P<0.01$ and $* * * P<0.001$

$\beta$ transgenic mice to investigate oestrogen receptor $\beta$-mediated cardioprotection $[9,14]$. Nonetheless, transgenic technology is far from clinical reality. Furthermore, in our experiments, no change was observed in the expression of cardiac oestrogen receptor $\beta$ either before or after MI induction. This phenomenon may indicate that the specific and potent activation of oestrogen receptor $\beta$, rather than a simple increase in the number of its molecules, is what is required for the treatment of MI injury. In recent years, with the technological development of the synthesis of chemical compounds, many studies have used DPN as a specific oestrogen receptor $\beta$ agonist $[13,26]$. Therefore, in the current study, we determined the role of oestrogen receptor $\beta$ in MI-induced cardiac injury by means of its specific agonist DPN. DPN treatment showed cardioprotective effects in our MI model, as evidenced by the attenuation of the following: increased oxidative stress, the increased number of apoptotic cardiomyocytes, myocardial enzyme release and the expansion of the infarct and fibrotic areas. It also enhanced myocardial angiogenesis and post-ischaemic cardiac functional recovery.

Although more and more data have proven the protective action of oestrogen receptor $\beta$ on the cardiovascular system, the underlying mechanisms remain to be further studied. 
Fig. 6 Effects of 2,3-bis(4hydroxyphenyl)-propionitrile (DPN) and $N-[N-(3,5-$ difluorophenacetyl)-1-alanyl]-sphenylglycine $t$-butyl ester (DAPT) on cardiac fibrosis after MI. a Haematoxylin and eosin (H\&E), Masson's trichrome and Sirius Red staining of transverse frozen tissue sections from each group 28 days after MI. b Statistical analysis of H\&E staining data (the average thickness of the left ventricles). c Statistical analysis of Masson's trichrome and Sirius Red staining (scar size). The values are the means \pm SEM. $n=6 ; * P<0.05$, $* * P<0.01$ and $* * * P<0.001$ a

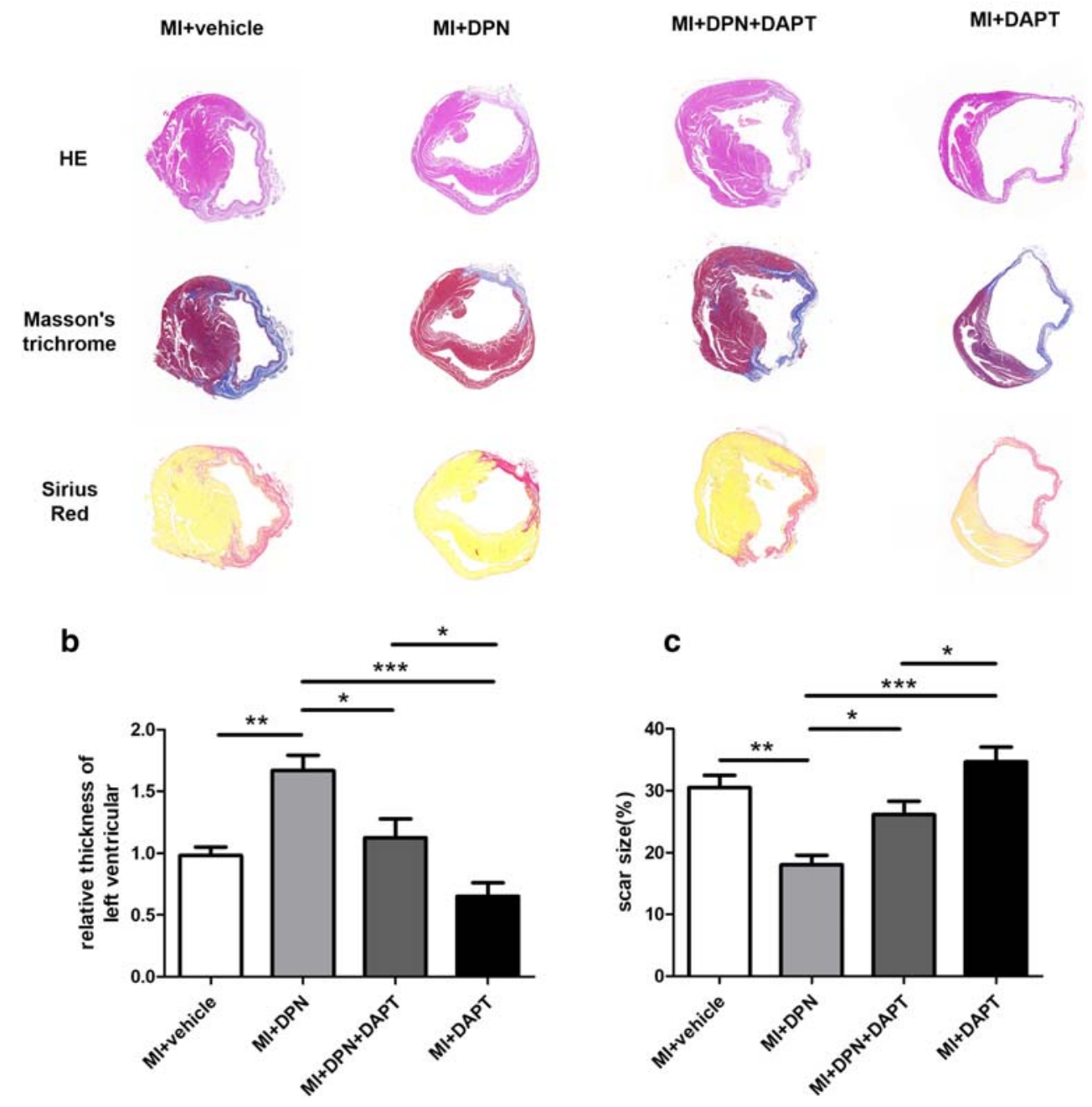

The Notch signalling pathway is highly conserved and controls cell fate throughout life. Recent studies have revealed that the Notch1 cascade performs a key function in the heart during development and disease [17]. Notch1 signalling is absent under normal conditions, but it can be activated in various tissues under pathological conditions, such as ischaemic injury and cancer $[38,39]$. One study has proven that Notch1 signalling exerts a regulatory effect during cardiac damage and preserves cardiac function after ischaemic insult [6]. Nevertheless, despite the advantages mentioned above, how exactly Notch1 exerts its protective effects remains unclear. In particular, we recently observed that Notch1 oestrogen receptor $\beta$ crosstalk is involved in the protection of the endothelium by oestrogen during TNF $\alpha$-induced inflammation [22]. Moreover, in the present study, we noted that NICD expression increased after MI injury and increased further after stimulation with DPN. Therefore, we propose that Notch1 signalling may play a key role in oestrogen receptor $\beta$-mediated cardioprotection after cardiac ischaemic damage.
DAPT, a specific inhibitor of $\gamma$-secretase, has been widely utilised to specifically block the Notch1 pathway by preventing NICD release [40]. Thus, to further elucidate the involvement of Notch1 signalling in the cardioprotective action of oestrogen receptor $\beta$, we decided to test its specific inhibitor DAPT. As a result, we observed that DAPT significantly attenuated the protective action of oestrogen receptor $\beta$ against MI, as evidenced by impaired recovery of postischaemic cardiac function, a reversal of the decrease in the levels of myocardial enzymes in the serum, the prevention of myocardial angiogenesis and the restoration of myocardial oxidation, apoptotic stress and fibrosis. Therefore, we seem to be the first to report that the upregulation of NICD can at least partially explain the beneficial effects of oestrogen receptor $\beta$ activation in our experimental settings.

To further characterise the role of Notch1 signalling in the cardioprotective activity of oestrogen receptor $\beta$, we decided to identify possible downstream signalling pathways. The $\mathrm{PI} 3 \mathrm{~K} / \mathrm{Akt}$ signalling pathway is well-known as a survival- 
Fig. 7 Effects of 2,3-bis(4hydroxyphenyl)-propionitrile (DPN) and $N-[N-(3,5-$ difluorophenacetyl)-1-alanyl]-sphenylglycine $t$-butyl ester

(DAPT) on the activation of the phosphatidylinositol-3-kinase (PI3K)/Akt pathway. a Western blot analysis of the expression of the intracellular domain of Notch (NICD) and of PI3K and Akt phosphorylation. $\mathbf{b}-\mathbf{f}$ Analysis of the levels of NICD, p-PI3K, pAkt, PI3K and Akt. The values are the means \pm SEM. $n=3$; $* * P<0.01$ and $* * * P<0.001$ a

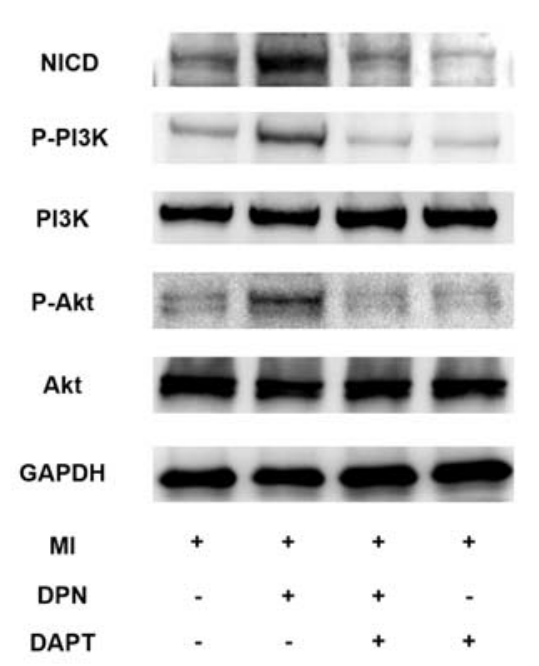

C

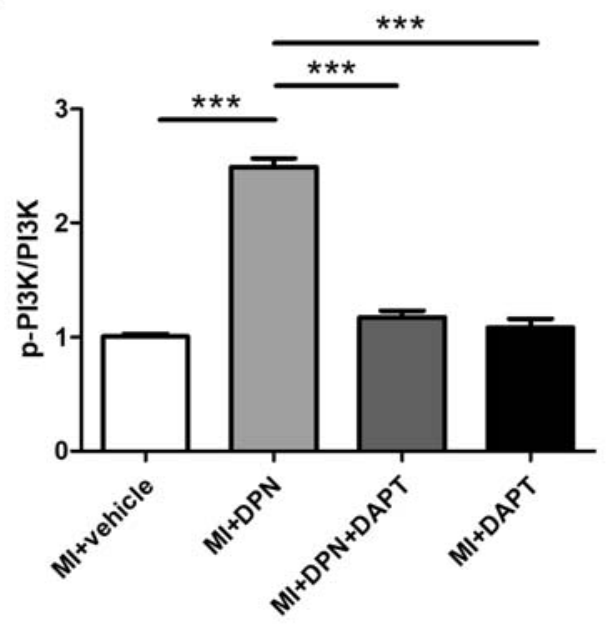

e

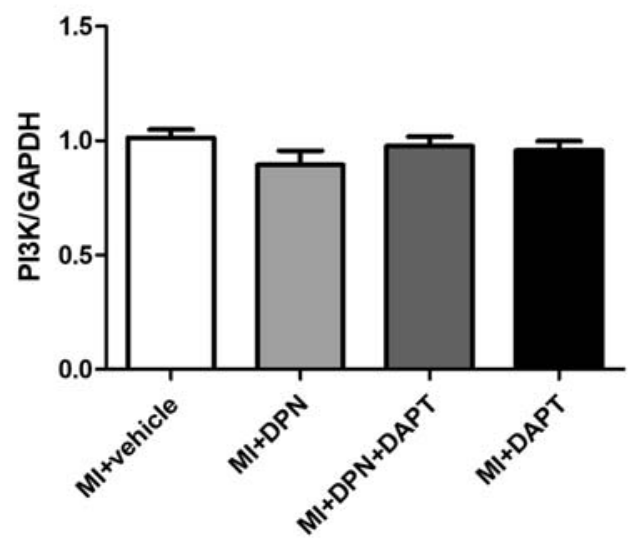

b

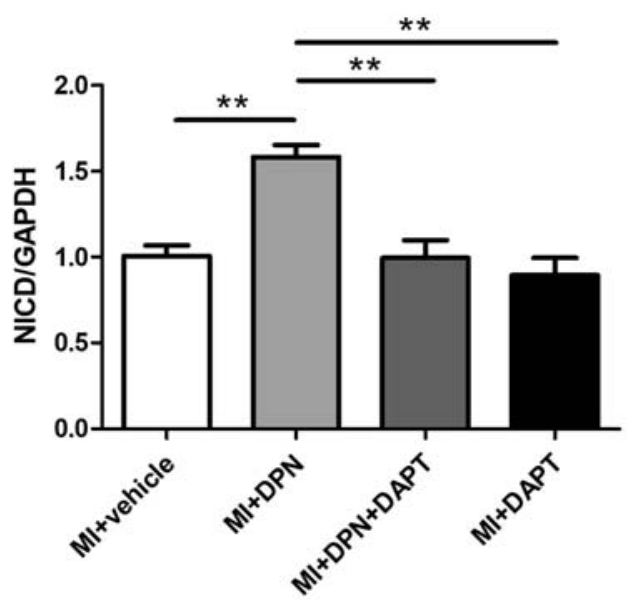

d
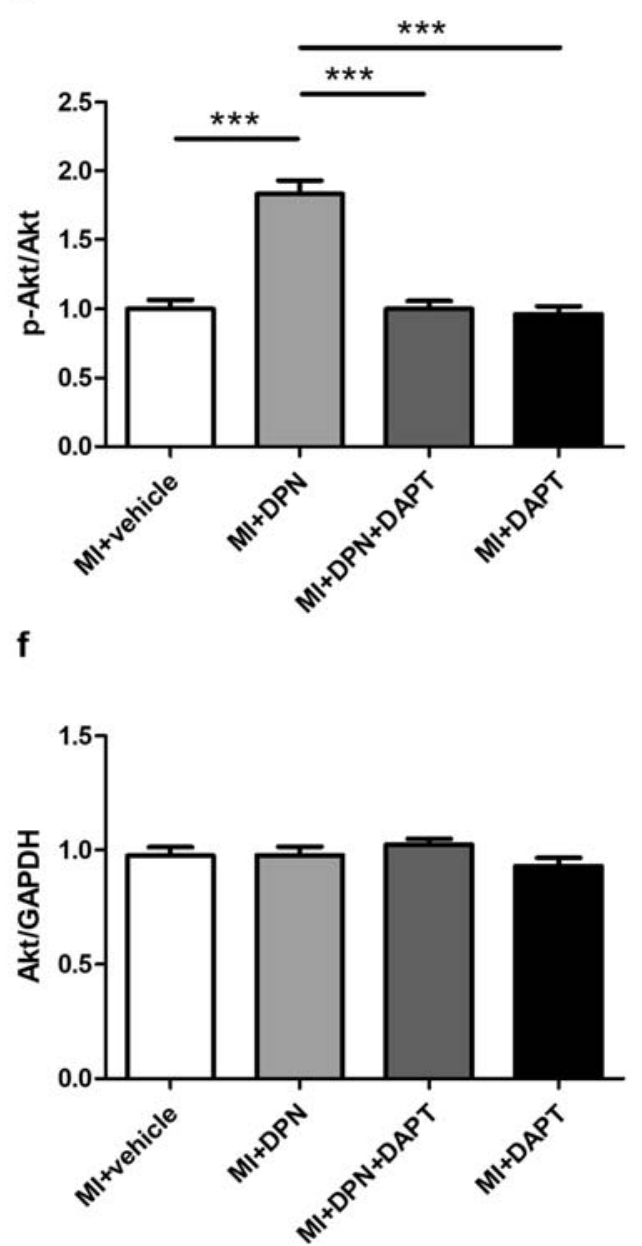

related signal transduction pathway that protects the myocardium from ischaemic injury [41]. In addition, it has been proven that the PI3K/Akt signalling pathway is activated in response to the chronic upregulation of oestrogen receptor $\beta$
[42]. Furthermore, Notch1 has been demonstrated to modulate the PI3K/Akt axis by regulating PTEN expression in various tissues [43]. These observations prompted us to investigate the relationship between Notch1, PI3K/Akt signalling and 


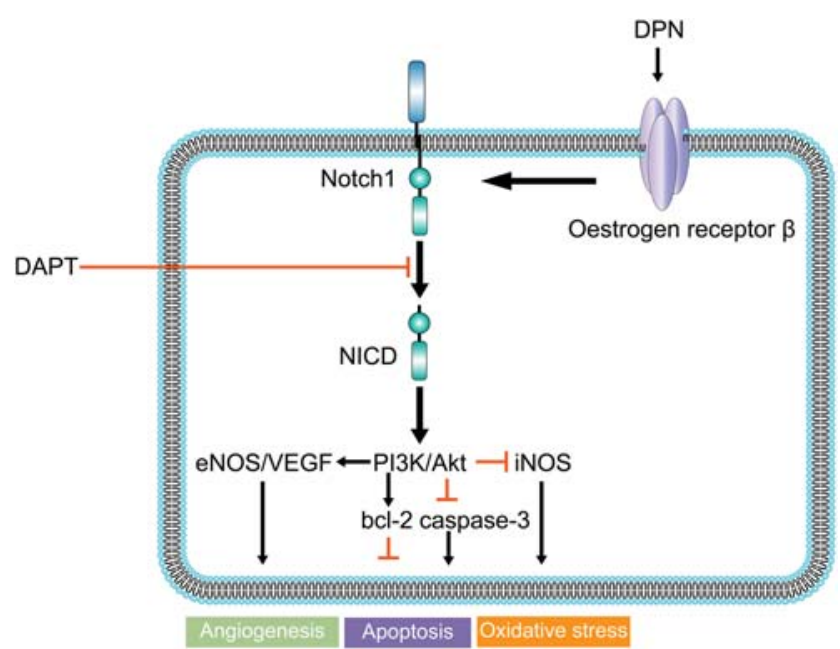

Fig. 8 Representative scheme depicting the possible mechanisms of oestrogen receptor $\beta /$ Notch1 signalling in protection against myocardial infarction injury. Direct oestrogen receptor $\beta$ stimulation by 2,3-bis(4hydroxyphenyl)-propionitrile (DPN) promotes the expression of intracellular domain of Notch1 (NICD) and protects the heart against oxidative stress, cardiomyocyte apoptosis and cardiac fibrosis. PI3K/ Akt signalling is the downstream effector of this protective action. Notch1 signalling inhibitor N-[N-(3,5-difluorophenacetyl)-L-alanyl]-Sphenylglycine t-butyl ester (DAPT) significantly attenuated the cardioprotective action of oestrogen receptor $\beta$

oestrogen receptor $\beta$ activation in damaged cardiac tissue. As a result, we revealed that oestrogen receptor $\beta$ activation significantly increased the expression of NICD and promoted the phosphorylation of proteins in the PI3K/Akt pathway. To elucidate the relationship between NICD expression and the PI3K/Akt pathway during oestrogen receptor $\beta$ activation, we blocked Notch1 signalling via its specific inhibitor DAPT. It is noteworthy that the phosphorylation of PI3K and Akt was effectively suppressed by the inhibition of Notch1 signalling regardless of whether DPN was administered. Therefore, we determined the relationship between oestrogen receptor $\beta$, Notch1 and the PI3K/Akt signalling cascades by confirming that the cardioprotective influence of oestrogen receptor $\beta$ is largely due to its ability to activate Notch1 signal transduction (i.e. NICD release and translocation to the nucleus) and subsequently, to enhance the PI3K/ Akt pathway.

\section{Conclusion}

The findings of our experiments support our hypothesis regarding oestrogen receptor $\beta /$ Notch1 signalling and suggest that oestrogen receptor $\beta$ stimulation by DPN activates Notch1 signalling and exerts profound cardioprotective effects against MI. In addition, PI3K/Akt signalling may be a downstream effector of this protective action. These findings provide new insight into the molecular mechanisms of the cardioprotective action of oestrogen receptor $\beta$ and may pave the way for the future application of oestrogen receptor $\beta$ agonists in the treatment of MI.

Funding Information This study was funded by the National Natural Science Foundation of China (grant no. 81670225), the Clinical Transformation Foundation of Shanghai Shenkang Hospital Development Center (grant no. 16CR3086B) and the Renji Hospital Pei Yu Foundation (grant no. PYIII-17-031).

\section{Compliance with Ethical Standards}

Conflict of Interest The authors declare that they have no conflicts of interest.

Ethical Approval All applicable international, national and/or institutional guidelines for the care and use of animals were followed.

\section{References}

1. Jessup M, Brozena S. Heart failure. N Engl J Med. 2003;348(20): 2007-18.

2. Jneid H, Fonarow GC, Cannon CP, Palacios IF, Kilic T, Moukarbel $\mathrm{GV}$, et al. Impact of time of presentation on the care and outcomes of acute myocardial infarction. Circulation. 2008;117(19):2502-9.

3. Piro M, Della Bona R, Abbate A, Biasucci LM, Crea F. Sex-related differences in myocardial remodeling. J Am Coll Cardiol. 2010;55(11):1057-65.

4. O'Meara E, Clayton T, McEntegart MB, McMurray JJ, Pina IL, Granger CB, et al. Sex differences in clinical characteristics and prognosis in a broad spectrum of patients with heart failure: results of the Candesartan in Heart failure: Assessment of Reduction in Mortality and morbidity (CHARM) program. Circulation. 2007;115(24):3111-20.

5. Dworatzek E, Mahmoodzadeh S. Targeted basic research to highlight the role of estrogen and estrogen receptors in the cardiovascular system. Pharmacol Res. 2017;119:27-35.

6. Rocca C, Femmino S, Aquila G, Granieri MC, De Francesco EM, Pasqua T, et al. Notch1 mediates preconditioning protection induced by GPER in normotensive and hypertensive female rat hearts. Front Physiol. 2018;9(521).

7. Eyster KM. The estrogen receptors: an overview from different perspectives. Methods Mol Biol. 2016;3127-9_1.

8. Wang M, Wang Y, Weil B, Abarbanell A, Herrmann J, Tan J, et al. Estrogen receptor beta mediates increased activation of PI3K/Akt signaling and improved myocardial function in female hearts following acute ischemia. Am J Physiol Regul Integr Comp Physiol. 2009;296(4):11.

9. Korte T, Fuchs M, Arkudas A, Geertz S, Meyer R, Gardiwal A, et al. Female mice lacking estrogen receptor beta display prolonged ventricular repolarization and reduced ventricular automaticity after myocardial infarction. Circulation. 2005;111(18):2282-90.

10. Schuster I, Mahmoodzadeh S, Dworatzek E, Jaisser F, Messaoudi $\mathrm{S}$, Morano I, et al. Cardiomyocyte-specific overexpression of oestrogen receptor beta improves survival and cardiac function after myocardial infarction in female and male mice. Clin Sci. 2016;130(5):365-76.

11. Lin J, Steenbergen C, Murphy E, Sun J. Estrogen receptor-beta activation results in S-nitrosylation of proteins involved in cardioprotection. Circulation. 2009;120(3):245-54.

12. Bulut EC, Abueid L, Ercan F, Suleymanoglu S, Agirbasli M, Yegen BC. Treatment with oestrogen-receptor agonists or oxytocin in 
conjunction with exercise protects against myocardial infarction in ovariectomized rats. Exp Physiol. 2016;101(5):612-27.

13. Nikolic I, Liu D, Bell JA, Collins J, Steenbergen C, Murphy E. Treatment with an estrogen receptor-beta-selective agonist is cardioprotective. J Mol Cell Cardiol. 2007;42(4):769-80.

14. Hamada H, Kim MK, Iwakura A, Ii M, Thorne T, Qin G, et al. Estrogen receptors alpha and beta mediate contribution of bone marrow-derived endothelial progenitor cells to functional recovery after myocardial infarction. Circulation. 2006;114(21):2261-70.

15. Xiang F, Shi Z, Guo X, Qiu Z, Chen X, Huang F, et al. Proteomic analysis of myocardial tissue from the border zone during early stage post-infarct remodelling in rats. Eur J Heart Fail. 2011;13(3):254-63.

16. Guruharsha KG, Kankel MW, Artavanis-Tsakonas S. The Notch signalling system: recent insights into the complexity of a conserved pathway. Nat Rev Genet. 2012;13(9):654-66.

17. de la Pompa JL, Epstein JA. Coordinating tissue interactions: Notch signaling in cardiac development and disease. Dev Cell. 2012;22(2):244-54.

18. Cheng Y, Gu W, Zhang G, Li X, Guo X. Activation of Notch1 signaling alleviates dysfunction of bone marrow-derived mesenchymal stem cells induced by cigarette smoke extract. Int J Chron Obstruct Pulmon Dis. 2017;12:3133-47.

19. Breunig JJ, Silbereis J, Vaccarino FM, Sestan N, Rakic P. Notch regulates cell fate and dendrite morphology of newborn neurons in the postnatal dentate gyrus. Proc Natl Acad Sci U S A. 2007;104(51):20558-63.

20. Li Y, Hiroi Y, Ngoy S, Okamoto R, Noma K, Wang CY, et al. Notch1 in bone marrow-derived cells mediates cardiac repair after myocardial infarction. Circulation. 2011;123(8):866-76.

21. Luxan G, Casanova JC, Martinez-Poveda B, Prados B, D'Amato G, MacGrogan D, et al. Mutations in the NOTCH pathway regulator MIB1 cause left ventricular noncompaction cardiomyopathy. Nat Med. 2013;19(2):193-201.

22. Fortini F, Vieceli Dalla Sega F, Caliceti C, Aquila G, Pannella M, Pannuti A, et al. Estrogen receptor beta-dependent Notch1 activation protects vascular endothelium against tumor necrosis factor alpha (TNFalpha)-induced apoptosis. J Biol Chem. 2017;292(44): 18178-91.

23. Gladyshev VN, Arner ES, Berry MJ, Brigelius-Flohe R, Bruford EA, Burk RF, et al. Selenoprotein gene nomenclature. J Biol Chem. 2016;291(46):24036-40.

24. Gu J, Fan Y, Liu X, Zhou L, Cheng J, Cai R, et al. SENP1 protects against myocardial ischaemia/reperfusion injury via a HIF1alphadependent pathway. Cardiovasc Res. 2014;104(1):83-92.

25. Du M, Gu J, Wang J, Xue Y, Ma Y, Mo X, et al. Silk fibroin/poly(Llactic acid-co-epsilon-caprolactone) electrospun nanofibrous scaffolds exert a protective effect following myocardial infarction. Exp Ther Med. 2019;17(5):3989-98.

26. Iorga A, Umar S, Ruffenach G, Aryan L, Li J, Sharma S, et al. Estrogen rescues heart failure through estrogen receptor Beta activation. Biol Sex Differ. 2018;9(1):018-0206.

27. Cheng J, Koenig SN, Kuivaniemi HS, Garg V, Hans CP. Pharmacological inhibitor of notch signaling stabilizes the progression of small abdominal aortic aneurysm in a mouse model. J Am Heart Assoc. 2014;3(6):001064.

28. Wang X, Guo Z, Ding Z, Mehta JL. Inflammation, autophagy, and apoptosis after myocardial infarction. J Am Heart Assoc. 2018;7(9): 008024.
29. Du M, Schmull S, Zhang W, Wang C, Lian F, Chen Y, et al. C-kit(+ )AT2R(+) bone marrow mononuclear cell subset is a superior subset for cardiac protection after myocardial infarction. Stem Cells Int. 2016;4913515(10):27.

30. Teng F, Yin Y, Cui Y, Deng Y, Li D, Cho K, et al. Salvianolic acid A inhibits endothelial dysfunction and vascular remodeling in spontaneously hypertensive rats. Life Sci. 2016;144:86-93.

31. Pei H, Du J, Song X, He L, Zhang Y, Li X, et al. Melatonin prevents adverse myocardial infarction remodeling via Notch1/Mfn2 pathway. Free Radic Biol Med. 2016;97:408-17.

32. Rocca C, Boukhzar L, Granieri MC, Alsharif I, Mazza R, Lefranc $\mathrm{B}$, et al. A selenoprotein T-derived peptide protects the heart against ischaemia/reperfusion injury through inhibition of apoptosis and oxidative stress. Acta Physiol. 2018;223(4):17.

33. Rocca C, Pasqua T, Boukhzar L, Anouar Y, Angelone T. Progress in the emerging role of selenoproteins in cardiovascular disease: focus on endoplasmic reticulum-resident selenoproteins. Cell Mol Life Sci. 2019;76(20):3969-85.

34. Liang J, Shang Y. Estrogen and cancer. Annu Rev Physiol. 2013;75:225-40.

35. Xin HB, Senbonmatsu T, Cheng DS, Wang YX, Copello JA, Ji GJ, et al. Oestrogen protects FKBP12.6 null mice from cardiac hypertrophy. Nature. 2002;416(6878):334-8.

36. Weill A, Dalichampt M, Raguideau F, Ricordeau P, Blotiere PO, Rudant J, et al. Low dose oestrogen combined oral contraception and risk of pulmonary embolism, stroke, and myocardial infarction in five million French women: cohort study. Bmj. 2016;10(353).

37. Nuedling S, Karas RH, Mendelsohn ME, Katzenellenbogen JA, Katzenellenbogen BS, Meyer R, et al. Activation of estrogen receptor beta is a prerequisite for estrogen-dependent upregulation of nitric oxide synthases in neonatal rat cardiac myocytes. FEBS Lett. 2001;502(3):103-8.

38. Croquelois A, Domenighetti AA, Nemir M, Lepore M, RosenblattVelin N, Radtke F, et al. Control of the adaptive response of the heart to stress via the Notch1 receptor pathway. J Exp Med. 2008;205(13):3173-85.

39. Gude NA, Emmanuel G, Wu W, Cottage CT, Fischer K, Quijada P, et al. Activation of Notch-mediated protective signaling in the myocardium. Circ Res. 2008;102(9):1025-35.

40. Yu L, Liang H, Lu Z, Zhao G, Zhai M, Yang Y, et al. Membrane receptor-dependent Notch1/Hes1 activation by melatonin protects against myocardial ischemia-reperfusion injury: in vivo and in vitro studies. J Pineal Res. 2015;59(4):420-33.

41. Okumura H, Nagaya N, Itoh T, Okano I, Hino J, Mori K, et al. Adrenomedullin infusion attenuates myocardial ischemia/ reperfusion injury through the phosphatidylinositol 3-kinase/Aktdependent pathway. Circulation. 2004;109(2):242-8.

42. Deng X, Xing X, Sun G, Xu X, Wu H, Li G, et al. Guanxin danshen formulation protects against myocardial ischemia reperfusion injury-induced left ventricular remodeling by upregulating estrogen receptor beta. Front Pharmacol. 2017;8(777).

43. Pai P, Shibu MA, Chang RL, Yang JJ, Su CC, Lai CH, et al. ERbeta targets ZAK and attenuates cellular hypertrophy via SUMO-1 modification in H9c2 cells. J Cell Biochem. 2018;119(9):7855-64.

Publisher's Note Springer Nature remains neutral with regard to jurisdictional claims in published maps and institutional affiliations. 